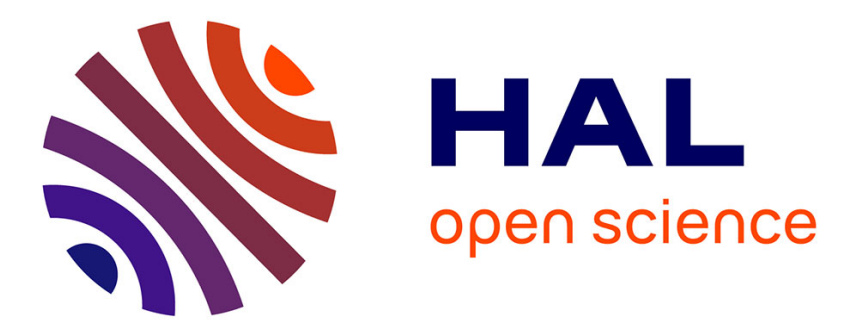

\title{
Slanted annular aperture arrays as enhanced-transmission metamaterials: Excitation of the plasmonic transverse electromagnetic guided mode
} Abdoulaye Ndao, Abderrahmane Belkhir, Roland Salut, Fadi Issam Baida

\section{To cite this version:}

Abdoulaye Ndao, Abderrahmane Belkhir, Roland Salut, Fadi Issam Baida. Slanted annular aperture arrays as enhanced-transmission metamaterials: Excitation of the plasmonic transverse electromagnetic guided mode. Applied Physics Letters, 2013, 103, pp.211901. 10.1063/1.4832227 . hal00924320

\section{HAL Id: hal-00924320 \\ https://hal.science/hal-00924320}

Submitted on 6 Jan 2014

HAL is a multi-disciplinary open access archive for the deposit and dissemination of scientific research documents, whether they are published or not. The documents may come from teaching and research institutions in France or abroad, or from public or private research centers.
L'archive ouverte pluridisciplinaire HAL, est destinée au dépôt et à la diffusion de documents scientifiques de niveau recherche, publiés ou non, émanant des établissements d'enseignement et de recherche français ou étrangers, des laboratoires publics ou privés. 


\title{
Slanted Annular Aperture Arrays as enhanced-transmission metamaterials: excitation of the plasmonic transverse electromagnetic guided mode
}

\author{
Abdoulaye Ndao ${ }^{1}$, Abderrahmane Belkhir ${ }^{2}$, Roland Salut ${ }^{1}$ and Fadi I. Baida ${ }^{1 *}$ \\ 1 Institut FEMTO-ST, UMR 6174 CNRS, \\ Département d'Optique P. M. Duffieux, \\ Université de Franche-Comté, \\ 25030 Besançon Cedex, France \\ 2 Université Mouloud Mammeri, \\ Laboratoire de Physique et Chimie Quantique, \\ Tizi-Ouzou, Algeria
}

\begin{abstract}
We present here the fabrication and the optical characterization of Slanted Annular Aperture Arrays (SAAA) engraved into silver film. An experimental enhanced transmission based on the excitation of the cutoff-less plasmonic guided mode of the nano-waveguides (the TEM mode) is demonstrated and agrees well with the theoretical predicted results. By the way, even if it is less efficient $(70 \% \rightarrow 20 \%)$, an enhanced transmission can occur at larger wavelength value $(720 \mathrm{~nm} \rightarrow 930 \mathrm{~nm})$ compared to conventional AAA structure by correctly setting the metal thickness.
\end{abstract}

Enhanced Optical Transmission (EOT) through arrays of subwavelength holes, drilled in optically thick metal films, has got a great deal of attention due both to its fundamental character and to potential applications such as flat optics [1], nanolithography [2], solar cells [3], fluorescence [4], chemical sensors [5], spectral filters [6], optical trapping [7], biomedical detection [8], second harmonic generation [9]... Many configurations have theoretically been proposed and experimentally built to demonstrate efficient EOT. A dual origin is generally behind this phenomenon: horizontal surface plasmon resonance that couples the apertures through the metal interface [10] or vertical plasmonic guided modes that allow obtaining higher transmission at larger value of wavelengths $[11,12]$.

Annular Aperture Arrays (AAA) are enhanced-transmission metamaterials where guided modes are directly involved [13]. In addition to the simple excitation of the $\mathrm{TE}_{11}$ mode that allows EOT up to $90 \%$ at normal incidence [14], the specificity of these apertures is that they possess a plasmonic guided mode without cutoff [15]. Nevertheless, the excitation of the latter[15-18] is subjected to

\footnotetext{
* Corresponding author: fbaida@univ-fcomte.fr, phone : 00(33) 381666421
} 


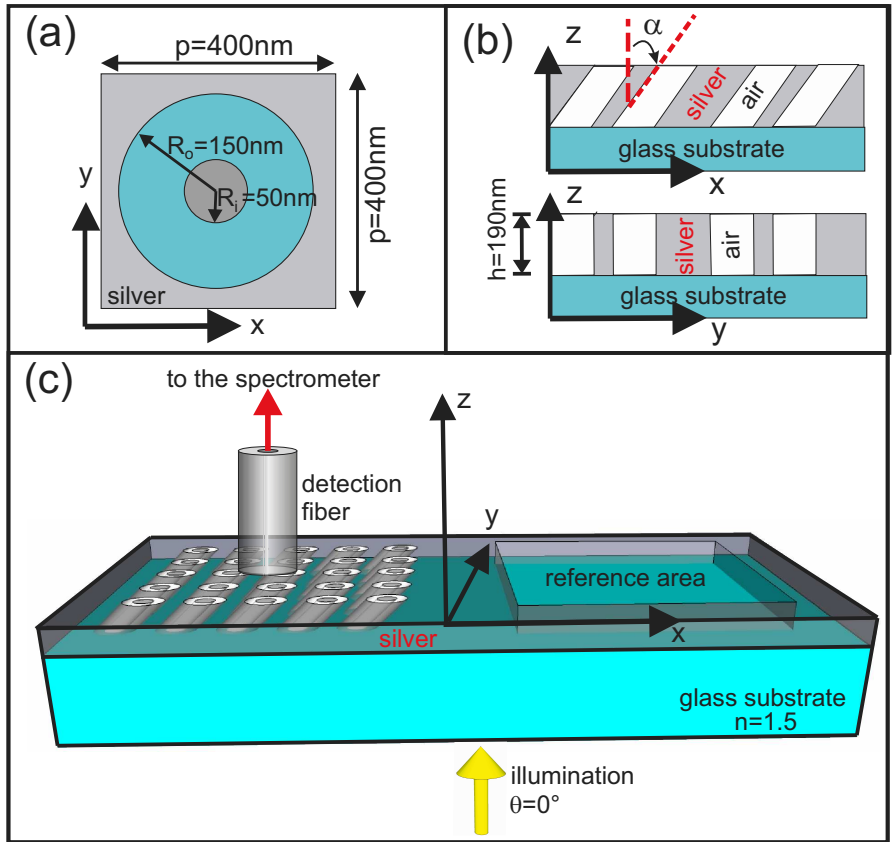

FIG. 1: a) Schematic of one annular aperture section in the $x O y$ plane giving the geometrical parameters (outer radius $R_{o}=150 \mathrm{~nm}$, inner radius $R_{i}=50 \mathrm{~nm}$ and period $p=400 \mathrm{~nm}$ ). b) Schema of the $x O z$ and $y O z$ sections showing the metallic film with thickness $h=190 \mathrm{~nm}$ perforated with SAAA. The tilted angle $\alpha=30^{\circ}$ of the aperture axis belongs to the $x O z$ plane. c) Sketch of the whole sample with the SAAA structure and the reference zone used to normalized the transmitted signal.

conditions that are sometimes experimentally difficult to be fulfilled such as the oblique incidence [17]. However, to bypass this constraint, an alternative solution was proposed by J.-J. Greffet team [19] and consists to incline the apertures instead of tilting the whole structure and/or the incident beam. Even if this solution seems easy in the domain of radio and military frequencies (millimeter or centimeter structure dimensions), we are clearly facing a real technological challenge which is to attempt scaling the geometrical parameters in order to operate in the visible domain. A first pure theoretical study of such structures was performed [20] to demonstrate the potential of these Slanted Annular Aperture Arrays (SAAA). The TEM-based enhanced transmission was numerically predicted for several configurations.

Here, a realistic structure is theoretically and experimentally studied after it is fabricated. The excitation of the TEM mode is demonstrated though the emergence of an additional transmission peak located at wavelength value larger than the one associated with the $\mathrm{TE}_{11}$ one as expected from the theoretical results. The aim of this study is to demonstrate the TEM-based enhancement in the transmission and does not deal with the excitation of the $\mathrm{TE}_{11}$ mode that was already 
discussed in several previous papers (see [12] and references therein).

Our structure (see Fig. 1) consists on slanted annular aperture array (SAAA) engraved into $h=190 \mathrm{~nm}$ thick silver film. This large metal thickness value is needed to set the TEM-based transmission peak beyond the $\mathrm{TE}_{11}$-based one. In fact, unlike the latter which is located around its cutoff wavelength, the position of the TEM peak is only given by a phase matching condition similar to the one of a Fabry-Perot interferometer [12]. Otherwise, the array period is chosen in order to push away both Wood's and Rayleigh's anomalies from the spectral range where enhanced transmission is expected especially for the TEM guided mode. Under these conditions, and in accordance with technological constraints of manufacturing, one fixes the SAAA structure parameters as given on figures $1 \mathrm{a}$ and $\mathrm{c}$.

Within these geometrical parameters (radii value), dispersion of the $\mathrm{TE}_{11}$ and the TEM mode is calculated for two different kind of metals: silver and gold (see figure 2). These calculations are done using a N-order FDTD code [21] that was adapted to accurately describe the metal dispersion properties by integrating the Drude-critical points model $[22,23]$ allowing to consider the metal dispersion from experimental data [24]. The effective index, real and imaginary parts, of the two guided modes is then deduced from the calculated electromagnetic spectral density. As mentioned in refs [11, 25], figure 2 confirms that the $\mathrm{TE}_{11}$ mode has a hybrid (conventional and plasmonic) behavior while the TEM mode is purely plasmonic one. According to the results of figure $2 \mathrm{~d}$, silver is chosen, instead of gold, to reduce the propagation losses and to reach efficient TEM-based transmission.

To achieve the fabrication of this structure, it was necessary to perform tests to adjust the settings of the used FIB (Dual Beam FEI Helios 600i) milling that is combined with a very accurate metal deposition process. A thin chromium layer $(5 \mathrm{~nm})$ is deposited as an adhesion layer on the glass substrate $(n=1.5)$. Next, a silver film (thickness $h=190 \mathrm{~nm}$ ) is deposited by evaporation and the SAAA grating is obtained by FIB milling of the metallic layer. The FIB operated at $30 \mathrm{keV}$, and the probe current is $1.1 \mathrm{pA}$. These parameters lead to an ion beam diameter around $5 \mathrm{~nm}$. Scanning electron microscope (SEM) images, shown in Fig. 3, allow us to check the quality and the geometrical parameters of the samples in comparison with the theoretical specifications. The tilt angle of the slanted apertures is set to $\alpha=30^{\circ}$ in the $x O z$ plane (angle between the annular aperture axis and the perpendicular to the metal film as shown in Fig. 1b). Fig. 3a is a top view SEM image of the whole fabricated array i.e. a matrix of $20 \times 20$ annular apertures. Fig. 3b is a SEM view in the $O y$ direction that clearly shows the slanted apertures while Fig. 3c is taken in the $O x$ direction. 

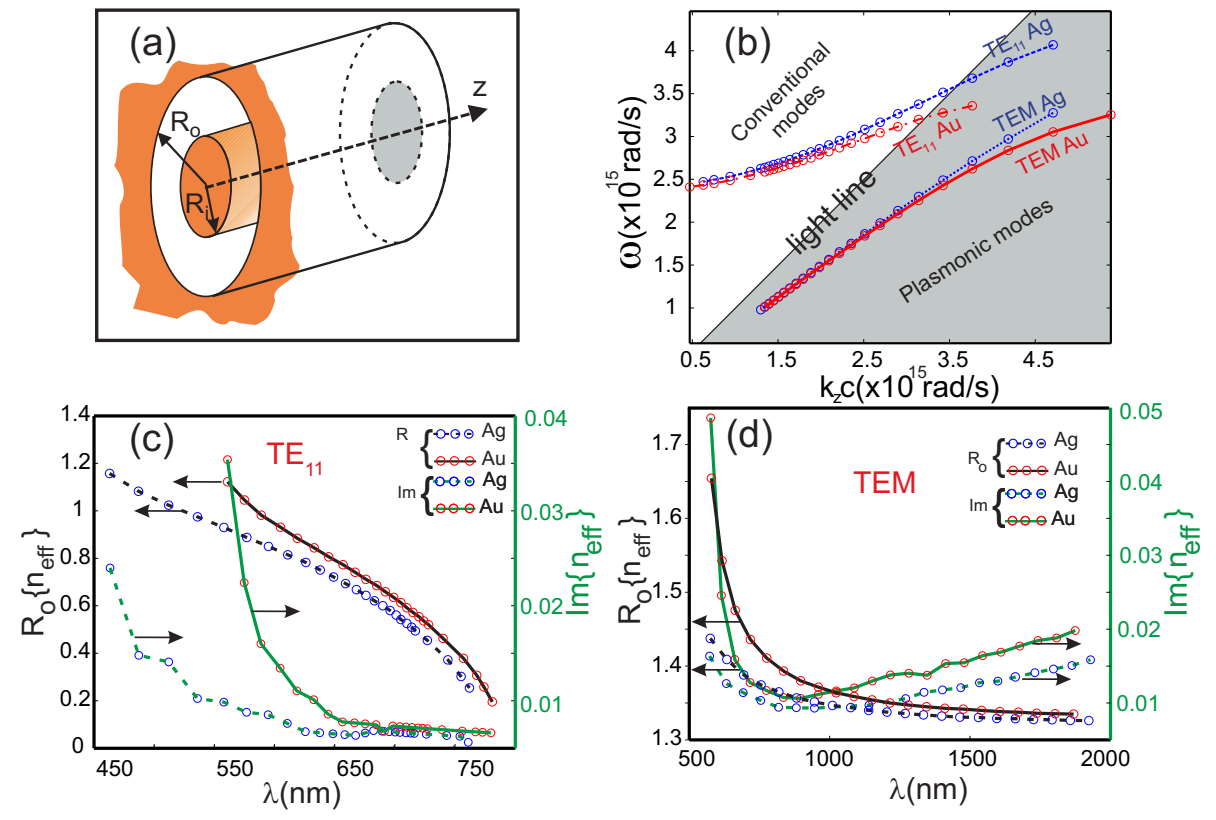

FIG. 2: (a) Schematic of the infinite coaxial waveguide considered for the determination of the real and imaginary part of the effective index and the dispersion diagram of the $\mathrm{TE}_{11}$ and the TEM guided modes. (b) Dispersion curves for waveguides made in gold (red lines) or silver (blue lines) when $R_{i}=50 \mathrm{~nm}$ and $R_{o}=150 \mathrm{~nm}$. (c) Real part (black curves: Ag in dashed and Au in solid lines) and imaginary part (green curves: $\mathrm{Ag}$ in dashed and $\mathrm{Au}$ in solid lines) of the effective index of the $\mathrm{TE}_{11}$ mode calculated from (b). (d) corresponding curves as in (c) for the TEM mode.

In order to check the fabrication quality of the apertures along the metal thickness, we have made a vertical cutting of the structure parallel to the $x O z$ plane passing through the centers of the apertures. For this purpose and to prevent the destruction of the core metallic parts, we have deposited a protective platinum layer on the surface of the SAAA to entirely fill the air gap inside the apertures. Platinum is deposited thanks to a gas injection system that brings the organometallic precursor very close to the sample. Platinum is widely used in FIB process because it is easy and fast to be deposited even with the electron beam avoiding destruction of the sample surface. Moreover, it allows good contrast with silver in SEM images due to the difference in their electronic properties. With simultaneously coupled FIB and SEM techniques, the cutting is observed in real time. Nevertheless, to optimize the FIB milling, the surface of the sample is set perpendicular to the ion column while the electron beam is tilted by $52^{\circ}$ with respect to the ion column.

The obtained SEM image is presented in Fig. 3d where the limitations of the used fabrication technique are patently seen. In fact, even if the average value of the tilt angle $\left(\alpha=30^{\circ}\right)$ is observed, 


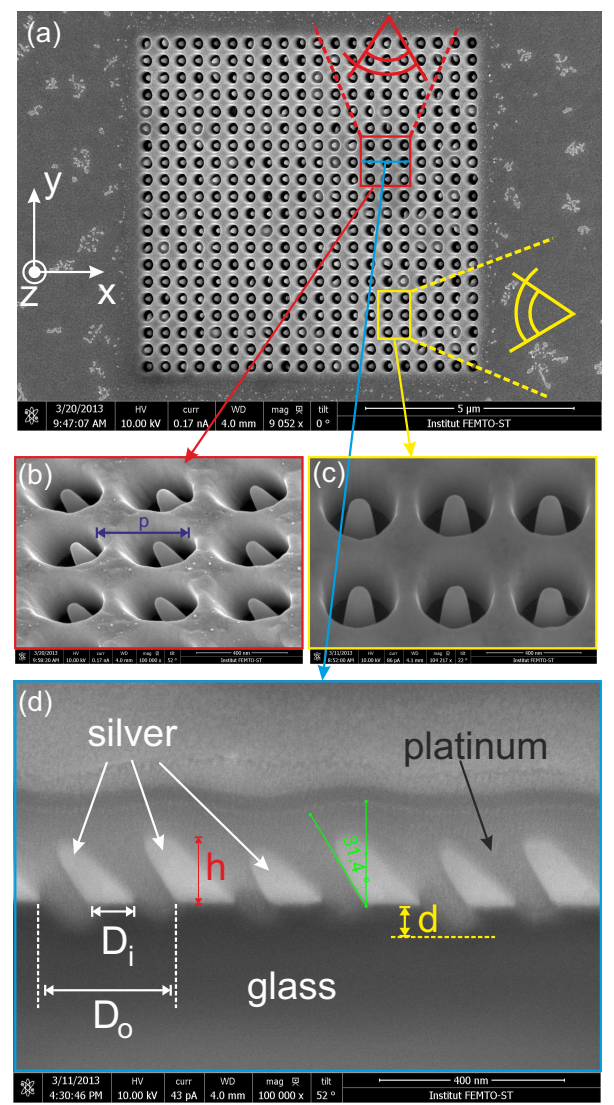

FIG. 3: (a) Top view SEM image of the studied SAAA matrix engraved in silver film. (b) is a zoom-in made over $3 \times 3$ patterns imaged in the $O y$ direction to point out the slanted apertures. (c) is a zoom-in made over $3 \times 2$ apertures in the $O x$ direction and (d) is a SEM image of a vertical section along the axis of the apertures after the apertures were filled by platinum. The measured average tilt angle $\alpha=31.4^{\circ}$, the inner diameter $D_{i}=95 \mathrm{~nm}$, and the outer diameter $D_{o}=295 \mathrm{~nm}$ are slightly different from the specified theoretical values given in Fig. 1 .

the inner and outer radii seem to vary with the vertical position along the metal thickness. This well-known effect is due to the FIB technique that allows conical shapes instead of regular vertical edges [26]. In addition, one can see that during the FIB milling of the apertures, a small part of the substrate is also engraved over almost $30-50 \mathrm{~nm}$. These manufacturing defects can affect the transmission properties of the SAAA and are probably the origin of the weak discrepancy between theoretical and experimental results.

The experimental transmission spectra are recorded as depicted in Fig. 1c. A powerful whitelight source (Leukos-SM-OEM) is used to illuminate the sample at normal incidence after it is collimated and linearly polarized. The light transmitted through the structure is collected by a cleaved-end multimode fiber (core diameter $62.5 \mu \mathrm{m}$ ). The collection fiber end is positioned in front 
of the sample by means of micro position stages, while the other end is connected to an optical spectrometer (USB2000 by Ocean Optics). As for the theoretical simulations, the experimental transmission spectrum is defined as the ratio between the intensity measured through the structure to the same quantity measured through a reference sample which consists on a squared hole made in the same silver film and with the same area as the whole SAAA.

The transmission spectra depicted in Fig. 4 exhibit a good agreement between theory and experiment. The $\mathrm{TE}_{11}$ guided mode is efficiently excited and transmission as large as $70 \%$ is obtained around its cutoff $\left(\lambda_{c}^{T E_{11}} \simeq 707 \mathrm{~nm}\right)$. Indeed, this transmission value is less than the one obtained by Poujet et al.[14] because the metal thickness, i.e. the propagation distance, is here larger $(h=190 \mathrm{~nm}$ instead of $100 \mathrm{~nm})$. In addition, further transmission peak occurs at $\lambda^{T E M} \simeq 935 \mathrm{~nm}$ that is due to the excitation of the TEM guided mode inside the apertures. Its amplitude only reaches $20 \%$ (see Fig. 4a) instead of $40 \%$ theoretically expected as shown on Fig. 4b). Nevertheless, the theoretical and experimental spectra are qualitatively very similar even if the some details are somewhat different due to the technological artifacts of fabrication. In fact,when looking closely to Fig. 3a, one can see that 78 apertures among the 400 that the SAAA contains, are almost not completely opened. This obviously leads to cancel the TEM guided mode excitation inside these apertures and affects the transmission amplitude of the TEM peak. Note here that theoretical simulations are performed by taking into account the engraved substrate over $d=37 \mathrm{~nm}$ and also by shortening the core metallic part by $20 \mathrm{~nm}$. Nonetheless, the conical effect was not considered.

In summary, we demonstrate the first challenging fabrication and experimental characterization of SAAA in the visible domain. A transmission peak due to the excitation of the TEM guided mode is also demonstrated. Even if the fabrication process has to be improved to fulfill the theoretical specifications, the spectral response of the SAAA is almost unaffected. The fabrication of such structure opens the way to the conception of a new generation of metamaterials with very interesting transmission properties.

\section{acknowledgements}

The author would thank Maria-Pilar Bernal for constructive and useful discussions. This work is partially supported by the Labex ACTION, and the French network of technology platforms 

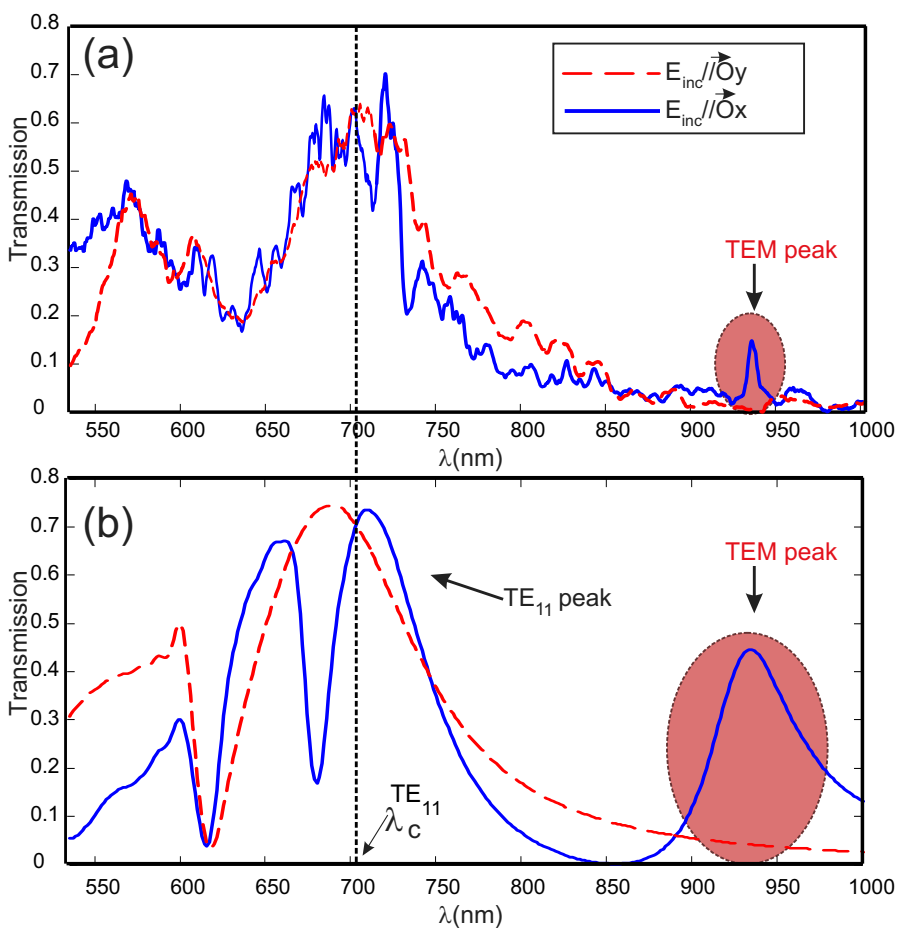

FIG. 4: Zero-order (a) experimental and (b) theoretical transmission spectra of the SAAA structure as a function of wavelength for the two cases of polarization: $E_{\text {inc }} / / O x$ (solid blue line) and $E_{\text {inc }} / / O y$ (dashed red line). The TEM guided mode is only excited when the incident electric field has a non-zero component along the aperture axis. Note that simulations are based on a home-made 3D FDTD code [27].

"Renatech".

[1] F. Aieta, P. Genevet, N. Yu, M. A. Kats, Z. Gaburro and F. Capasso, Nano Lett., 12, 1702-1706 (2012).

[2] S. Shinada, J. Hashizume and F. Koyama, Appl. Phys. Lett., 83, 836 (2003).

[3] L. Mo, L. Yang and S. He, ACP Technical Digest, OSA (2012).

[4] H. Rigneault and P.-F. Lenne, J. Opt. Soc. Am. B, 20, 2203 (2003).

[5] A. G. Brolo, R. Gordon, B. Leathem and K. Kavanagh, Langmuir 20, 4813-4815 (2004).

[6] S. Yokogawa, S. P. Burgos and H. A. Atwater, Nano Lett. 12, 4349-4354 (2012).

[7] A. A. E. Saleh and J. A. Dionne, Nano Lett. 12, 5581-5586 (2012).

[8] F. Ye, M. J. Burns and M. J. Naughton, Nano Lett. 13, 519-523, 2013.

[9] E. Barakat, M.-P. Bernal and F. I. Baida, Opt. Express 20, 16258-16268 (2012).

[10] T. Ebbesen, H. Lezec, H. Ghaemi, T. Thio and P. Wolff, Nature 391, 667-669 (1998).

[11] F. I. Baida, A. Belkhir, D. Van Labeke and O. Lamrous, Phys. Rev. B 74, 205419 (2006). 
[12] F. Baida and J. Salvi, Ed. Chris D. Geddes, Springer, New York (2011).

[13] F. I. Baida, D. Van Labeke, G. Granet, A. Moreau and A. Belkhir, Appl. Phys. B 79, 1-8 (2004).

[14] Y. Poujet, J. Salvi and F. I. Baida, Opt. Lett. 32, 2942-2944 (2007).

[15] A. Moreau, G. Granet, F. I. Baida and D. Van Labeke, Opt. Express 11, 1131-1136 (2003).

[16] M. J. Lockyear, A. P. Hibbins, Ch. R. Lawrence and J. R. Sambles, Phys. Rev. Lett. 94, 193902 (2005).

[17] F. I. Baida, Appl. Phys. B 89, 145-149 (2007).

[18] A. Roberts, Opt. Express 18, 2528 (2010).

[19] S. Nosal, PhD thesis, Ecole Centrale Paris (2009).

[20] F. Baida, A. Belkhir, O. Arar, E. Barakat, J. Dahdah, C. Chemrouk, D. Van Labeke, C. Diebold, N. Perry and M.-P. Bernal, Micron 41, 742-745 (2010).

[21] C. T. Chan, Q. L. Yu and K. M. Ho, Phys. Rev. B 51, 16635 (1995).

[22] A. Vial and T. Laroche, J. Phys. D: Appl. Phys. 40, 7152-58 (2007).

[23] M. Hamidi, F. I. Baida, A. Belkhir and O. Lamrous, J. Phys. D: Appl. Phys. 44, 245101 (2011).

[24] E. Palik," Handbook of Optical Constants of Solids", Academic Press, London (1985).

[25] M. I. Haftel, C. Schlockermann and G. Blumberg, Phys. Rev. B 74, 235405 (2006).

[26] F. Lacour, N. Courjal, M.-P. Bernal, A. Sabac, C. Bainier, and M. Spajer, Optical Materials 27, 1421-1425 (2005).

[27] F. I. Baida and D. Van Labeke, Optics Commun. 209, 17-22 (2002). 